\title{
Contraceção em Mulheres com Enxaqueca: A Realidade de uma Consulta de Cefaleias
}

\section{Contraception in Women with Migraine: The Reality of a Headache Dedicated Consultation}

Daniela Sousa Coelho ${ }^{1, *}$, (D) Ivânia Alves ${ }^{2}$, (D) Sérgio Mouro Marcos ${ }^{3}$, Carla Fraga ${ }^{2}$

1-Unidade de Saúde Familiar Hygeia, Lixa, Portugal

2-Serviço de Neurologia / Centro Hospitalar do Tâmega e Sousa, Penafiel, Portugal

3-Serviço de Urgência / Hospital Senhora da Oliveira E.P.E. - Guimarães, Portugal

DOI: https://doi.org/10.46531/sinapse/AO/210013/2021

Informações/Informations: Artigo Original, publicado em Sinapse, Volume 21, Número 3, julho-setembro 2021. Versão eletrónica em www.sinapse.pt Original Article, published in Sinapse, Volume 21, Numbe

3, July-September 2021.

Electronic version in www. sinapse.pt

(C) Autor (es) (ou seu (s) empregador (es)) e Sinapse 2021. Reutilização permitida de acordo com CC BY-

NC. Nenhuma reutilização comercial.

(C) Author(s) (or their employer(s)) and Sinapse 2021.

Re-use permitted under CC BYNC. No commercial re-use.

Palavras-chave:

Anticoncepcionais Orais

Combinados:

Anticoncepcionais Orais

Hormonais

Perturbações de Enxaqueca.

Keywords:

Contraceptives, Oral

Combined;

Contraceptives, Oral,

Hormonal;

Migraine Disorders

\footnotetext{
*Autor Correspondente / Corresponding Author: Daniela Patrícia Sousa Coelho USF Hygeia

Rua António Nobre

4615-595 Lixa (Felgueiras), Portugal

eudanielasousacoelho@gmail. com
}

Recebido / Received: 2021-03-15 Aceite / Accepted: 2021-10-07 Publicado / Published: 2021-10-20

\section{Resumo}

Introdução: A enxaqueca é uma doença neurológica muito prevalente que está associada a doença cérebro-cardiovascular. Mulheres com história de enxaqueca que usam contraceção oral combinada têm maior risco de acidente vascular cerebral. Neste estudo pretendeu-se determinar a prevalência do uso dos diferentes métodos contracetivos nas mulheres em idade fértil, com diagnóstico de enxaqueca, seguidas em consulta hospitalar dedicada a cefaleias ao longo de 2019.

Material e Métodos: Foi realizado um estudo observacional descritivo. Incluíram-se todas as mulheres entre os 18 e os 54 anos (adultas em idade considerada fértil), com diagnóstico de enxaqueca, observadas na consulta externa de Neurologia/Cefaleias do Centro Hospitalar Tâmega e Sousa, em 2019. Os dados foram recolhidos, após assinatura do consentimento informado, mediante inquérito presencial (formulário) e posteriormente analisados.

Resultados: Incluíram-se 108 mulheres com enxaqueca. Da amostra, 52\% tinha como diagnóstico enxaqueca com aura. Os contracetivos orais combinados foram o método contracetivo mais frequentemente utilizado. Do total, cerca de 30,5\% das mulheres não cumpria as recomendações nacionais e internacionais, da Organização Mundial de Saúde, relativas ao uso de métodos contracetivos em mulheres com enxaqueca.

Discussão: Nesta amostra, a prevalência de enxaqueca sem aura foi de menos de $50 \%$ sendo que este facto poderá ser explicado por um viés de referenciação à consulta hospitalar. Verificou-se uma prevalência considerável de não cumprimento das recomendações nacionais e internacionais relativas à escolha do método contracetivo nesta população.

Conclusão: Devem ser reunidos esforços para aumentar o cumprimento das recomendações.

\section{Abstract}

Introduction: Migraine is a prevalent neurological disorder associated with cerebro and cardiovascular diseases. Women with migraine that are also under oral contraceptives are at greater risk for cerebrovascular accidents. With this study we intend to investigate the prevalence of each contraceptive method used by women, diagnosed with migraine, followed in an headache dedicated consultation.

Material and Methods: A descriptive observational study was conducted. All 
women between 18 and 54 years old (adult women of childbearing age), diagnosed with migraine, followed in the Neurology/Headache consultation from Centro Hospitalar do Tâmega e Sousa during 2019, were included. Data was collected by faceto-face survey after signature of informed consent.

Results: One hundred and eight patients were included. Of the sample, 52\% was diagnosed with migraine with aura. Oral combined contraceptives were the most used method. Of the sample, 30.5\% were not following national or international recommendations (World Health Organization) regarding the use of contraceptives in women with migraine.

Discussion: In this sample, the prevalence of migraine without aura, being under $50 \%$, may reflect a referral bias towards the dedicated headache consultation. Non abidance to national or international recommendations regarding the choice of contraceptive in woman with migraine was considerable.

Conclusion: Action must be taken in order to increase abidance to national and international recommendations.

\section{Introdução}

A enxaqueca afeta cerca de $15 \%$ da população mundial.'

Dados publicados em 1993 davam conta de que cerca de $12 \%$ de uma população de estudantes universitários em Portugal tinham enxaqueca $(6 \%$ desses estudantes tinham enxaqueca isolada e $6 \%$ tinham enxaqueca associada a outros tipos de cefaleia). ${ }^{2}$ Outro estudo realizado na população portuguesa estimou a prevalência de enxaqueca, ao longo da vida, em 16\% (contabilizando os casos em que existe apenas enxaqueca e os casos em que esta existe associada a outros tipos de cefaleia). ${ }^{3}$

A incidência desta cefaleia primária é maior no sexo feminino e aumenta após a puberdade, atingindo sobretudo mulheres em idade fértil. ${ }^{4}$

Ao longo dos anos, a enxaqueca tem sido associada a eventos vasculares, como o acidente vascular cerebral (AVC) e o enfarte agudo do miocárdio (EAM), sendo esta associação mais significativa em mulheres com enxaqueca com aura. ${ }^{5}$ Perante estes factos, a enxaqueca passou a integrar, desde 2017 e pela primeira vez, uma ferramenta usada na estratificação de risco para doença vascular. $^{6}$

Sabe-se que os contracetivos hormonais combinados ( $\mathrm{CHC}$ ) estão associados, por seu lado, a um pequeno aumento do risco de trombose venosa profunda, EAM e AVC sendo este risco maior em mulheres que têm múltiplos fatores de risco para doença vascular, de que é exemplo a enxaqueca com aura. ${ }^{7}$ Assim, mulheres com diagnóstico de enxaqueca que usam contraceção oral combinada (COC) têm cerca de $2-4$ vezes maior pro- babilidade de ter um AVC. ${ }^{8} \mathrm{~A} C \mathrm{COC}$ está contraindicada na mulher com enxaqueca com aura e na mulher com enxaqueca sem aura e $\geq 35$ anos de idade.., 10

Este trabalho teve como objetivo primário determinar a prevalência de uso de cada um dos métodos contracetivos nas mulheres em idade fértil, com diagnóstico de enxaqueca, seguidas na Consulta Externa de Neurologia/Cefaleias (CECef) do Centro Hospitalar Tâmega e Sousa (CHTS) ao longo de 2019. Desta forma pretendemos calcular a percentagem de cumprimento das recomendações nacionais e internacionais relativas ao uso de contraceção em mulheres com enxaqueca.

\section{Material e Métodos}

Foi realizado um estudo observacional descritivo.

\section{Colheita dos Dados}

Foi criado um formulário de inquérito com os dados a serem recolhidos, bem como um protocolo de investigação.

Após aprovação do protocolo pela Comissão de Ética do CHTS, foi proposta a participação no estudo a todas as mulheres entre os 18 os 54 anos, com diagnóstico de enxaqueca, observadas na CECef do CHTS, em 2019.

Uma revisão mostrou que a idade média da menopausa em mulheres de países industrializados varia entre 50 e 52 anos, existindo alguma evidência de que a idade da menopausa tem aumentado ao longo do tempo. " Em Portugal, de acordo com a Direção Geral da Saúde, a idade fértil da mulher assume-se até aos 54 anos, ${ }^{12}$ moti- 
vo pelo qual a participação no estudo foi proposta a este grupo de mulheres.

As mulheres que aceitaram participar no estudo, mediante assinatura de consentimento informado, responderam a um inquérito presencial (formulário), após a consulta.

Foram recolhidos os seguintes dados:

- Características clínicas da cefaleia (com ou sem aura, uso de medicação profilática, relação da enxaqueca com o cataménio);

- Idade;

- Dados antropométricos (peso e altura);

- Antecedentes médicos pessoais e familiares (história de diabetes, dislipidemia, hipertensão arterial, fibrilhação auricular, doença renal crónica, artrite reumatoide, lúpus e história familiar de angina ou enfarte do miocárdio em indivíduos com idade $<60$ anos);

- Método contracetivo em uso;

- Impressão subjetiva da mulher sobre o impacto (em termos de frequência e intensidade) dos vários contracetivos usados na evolução da enxaqueca.

\section{Objetivos primários}

- Determinar a prevalência de uso de cada um dos métodos contracetivos nas mulheres com enxaqueca seguidas na CECef do CHTS, em 2019.

- Determinar a percentagem de cumprimento/incumprimento das recomendações nacionais ${ }^{9}$ e internacionais $^{10}$ relativas ao uso de métodos contracetivos em mulheres com enxaqueca.

\section{Objetivos secundários}

- Correlacionar a proporção de doentes com necessidade de medicação profilática em cada subgrupo e o uso de métodos contracetivos.

- Conhecer a impressão subjetiva das mulheres acerca do impacto dos diferentes métodos contracetivos na evolução da enxaqueca.

\section{Estatística}

Os inquéritos foram sujeitos a uma anonimização. Após preenchimento de todos os inquéritos foi criada uma base de dados no programa SPSS ${ }^{\circledR}$ Versão 26. A análise dos dados passou por uma caracterização da população estudada em termos de média e/ou mediana das variáveis quantitativas e proporção das variáveis qualitativas. Para avaliar a normalidade da distribuição das variáveis quantitativas foram usados os testes de normalidade: Kolmogorov-Smirnov e Shapiro-Wilks. Para identificar diferenças entre os grupos realizaram-se testes paramétricos e não paramétricos (estes últimos para as variáveis com distribuição não normal) para as variáveis contínuas e teste qui-quadrado para as variáveis qualitativas. A análise de comparação/associação de variáveis nominais/categóricas entre mais do que 2 grupos foi realizada através da aplicação do teste de qui-quadrado às respetivas tabelas de contingência. Calcularam-se intervalos de confiança (IC) a $95 \%$ para as proporções encontradas.

\section{Resultados}

Participaram no estudo 108 mulheres com enxaqueca. Da amostra, 52\% tinha um diagnóstico de enxaqueca com aura $(n=56)$ enquanto os outros $48 \%$ da amostra eram constituídos por mulheres com o diagnóstico de enxaqueca sem aura. A proporção de mulheres com enxaqueca com e sem aura na amostra não foi significativamente diferente $(p=0, \mid 48)$.

A análise das variáveis idade e índice de massa corporal (IMC) mostrou que os resultados encontrados para as 108 mulheres não seguem uma distribuição normal ( $p<0,05$ para os testes de normalidade) ainda que quando se analise isoladamente os grupos de mulheres com enxaqueca com e sem aura, se verifique uma distribuição normal da idade ( $p=0,200$ em ambos os grupos). As mulheres, com enxaqueca com aura, incluídas no estudo, eram significativamente mais velhas que as mulheres com enxaqueca sem aura $(p<0,05)$. Não se verifica associação significativa entre o tipo de enxaqueca e o uso de medicação profilática, a predominância temporal das crises, o IMC, o tabagismo ou a presença de outros fatores de risco vascular (FRV) -Tabela 1.

\section{Métodos contracetivos utilizados}

$\mathrm{Na}$ amostra deste estudo, os COC foram o método contracetivo mais frequentemente utilizado (Fig. 1). Não se verificou associação entre o tipo de enxaqueca e o método contracetivo utilizado $(p=0,869)$, nem entre o método contracetivo e o uso de medicação profilática $(p=0,576)$. 
Tabela 1. Caracterização da amostra.

\begin{tabular}{|c|c|c|c|c|}
\hline Variável & $\begin{array}{c}\text { Total } \\
(n=108)\end{array}$ & $\underset{(n=56)}{\operatorname{COM} \text { aura }}$ & $\begin{array}{l}\text { SEM aura } \\
(n=52)\end{array}$ & $\begin{array}{c}p \\
\text { (Com vs. } \\
\text { Sem aura) }\end{array}$ \\
\hline Atualmente sob profilaxia & $67 \%(n=72)$ & $73 \%(n=41)$ & $60 \%(n=31)$ & 0,134 \\
\hline $\begin{array}{l}\text { Relação com o cataménio } \\
\text { - Crises apenas ou } \\
\text { predominantemente no cataménio } \\
\text { Crises em qualquer altura do ciclo } \\
\text { menstrual } \\
\text { - Crises predominantemente fora do } \\
\text { catamenio }\end{array}$ & $\begin{array}{c}18,5 \%(n=20) \\
76,9 \%(n=83) \\
4,6 \%(n=5)\end{array}$ & $\begin{array}{c}25 \%(n=14) \\
70 \%(n=39) \\
5 \%(n=3)\end{array}$ & $\begin{array}{c}11,5 \%(n=6) \\
84,6 \%(n=44) \\
3,8 \%(n=2)\end{array}$ & 0,169 \\
\hline $\begin{array}{l}\text { Idade em anos média } \pm \text { desvio padrão } \\
\text { mediana (valor mínimo; valor máximo) }\end{array}$ & $\begin{array}{l}36,6 \pm 9,357 \\
37(18 ; 54)\end{array}$ & $\begin{array}{c}38,9 \pm 8,43 \\
39,5(21 ; 54)\end{array}$ & $\begin{array}{l}34,1 \pm 9,738 \\
33,5(18 ; 52)\end{array}$ & 0,008 \\
\hline $\begin{array}{l}\text { IMC em kg/m² média } \pm \text { desvio padrão } \\
\text { mediana (valor mínimo; valor máximo) }\end{array}$ & $\begin{array}{l}25,7 \pm 4,285 \\
25,4(17 ; 41)\end{array}$ & $\begin{array}{l}25,7 \pm 4,099 \\
25,7(19 ; 41)\end{array}$ & $\begin{array}{l}25,6 \pm 4,514 \\
24,9(17 ; 37) \\
\end{array}$ & 0,453 \\
\hline $\begin{array}{l}\text { Tabagismo } \\
\text { · Não fumadora } \\
\text { · Ex-fumadora } \\
\text { · Fumadora }\end{array}$ & $\begin{array}{c}87 \%(n=94) \\
1,9 \%(n=2) \\
11,1 \%(n=12)\end{array}$ & $\begin{array}{c}83,9 \%(n=47) \\
1,8 \%(n=1) \\
14,3 \%(n=8)\end{array}$ & $\begin{array}{c}90,4 \%(n=47) \\
1,9 \%(n=1) \\
7,7 \%(n=4)\end{array}$ & 0,552 \\
\hline $\begin{array}{l}\text { Outros FRV } \\
\text { - Obesidade } \\
\text { - HTA } \\
\text { - Dislipidemia } \\
\text { - Familiar com doença cardíaca } \\
\text { isquémica < } 60 \text { anos } \\
\cdot>1 \text { destes FRV }\end{array}$ & $\begin{array}{c}30 \%(n=32) \\
15,7 \%(n=17) \\
5,6 \%(n=6) \\
4,6 \%(n=5) \\
16,7 \%(n=18) \\
2,8 \%(n=3)\end{array}$ & $\begin{array}{c}32 \%(n=18) \\
12,5 \%(n=7) \\
8,9 \%(n=5) \\
3,6 \%(n=2) \\
14,3 \%(n=8) \\
5,4 \%(n=3)\end{array}$ & $\begin{array}{c}27 \%(n=14) \\
19,2 \%(n=10) \\
1,9 \%(n=1) \\
5,8 \%(n=3) \\
19,2 \%(n=10) \\
0 \%(n=0)\end{array}$ & 0,128 \\
\hline
\end{tabular}

FRV - fatores de risco vascular; HTA - hipertensão arterial; IMC - índice de massa corporal.

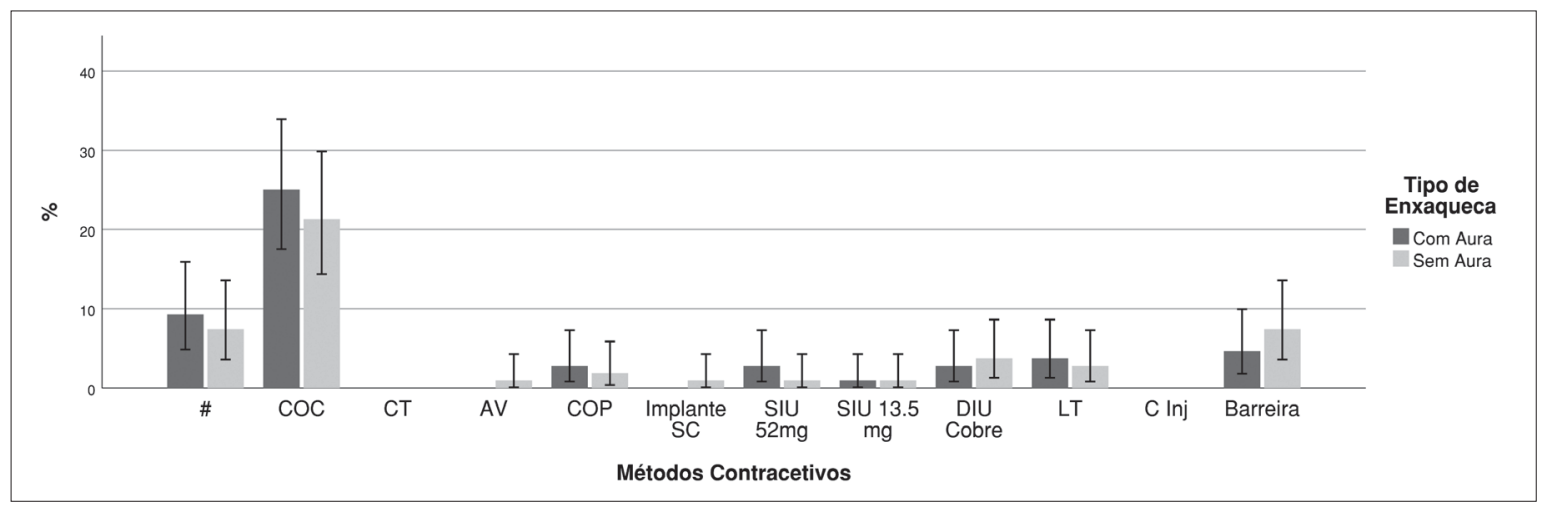

Figura 1. Distribuição dos métodos contracetivos nas mulheres com enxaqueca.

Barra de erro - intervalo de confiança a 95\%; \# - nenhum contracetivo em uso; AV - anel vaginal; Barreira - método de barreira; COC - contraceção oral combinada; COP - contraceção oral progestativa; CT - contraceção transcutânea; C Inj - contraceção injetável; DIU-cobre - dispositivo intra-uterino de cobre; LT - laqueação das trompas; SIU 52 mg - sistema intra-uterino com 52 mg de levonorgestrel; SIU 13,5 mg - sistema intra-uterino com 13,5 mg de levonorgestrel.

Como supracitado, das 108 participantes $52 \%$ tinha diagnóstico de enxaqueca com aura, das quais $48 \%$ (95\% intervalo de confiança (IC) $35 \%$ a $61 \%$ ) utilizavam COC. Das mulheres com enxaqueca sem aura mas com 35 ou mais anos de idade $(n=25), 24 \%$ (95\% IC 10\% a 43\%) utilizavam COC.

Portanto, da amostra, pelo menos $30,5 \%$ não cumpria com as recomendações nacionais ${ }^{9} \mathrm{e}$ internacionais ${ }^{10}$ acerca do uso de métodos contracetivos em doentes com enxaqueca.
Pela análise da Fig. 1 percebe-se ainda que 50,9\% (95\% IC 4 I\% a 60\%) das mulheres utiliza contraceção oral (a COC é utilizada por 27 mulheres com enxaqueca com aura e por 23 mulheres com enxaqueca sem aura e a contraceção oral progestativa (COP) é utilizada por 3 mulheres com enxaqueca com aura e 2 mulheres com enxaqueca sem aura). Verifica-se ainda que $16,7 \%$ (95\% IC I0\% a 24\%) da amostra $(n=18)$ não utiliza nenhum método de contraceção. 


\section{Uso de medicação profilática}

Setenta e duas mulheres usavam medicação profilática (67\% da amostra), das quais 63 usavam I fármaco e 9 usavam 2 fármacos. O fármaco profilático mais comumente utilizado foi o topiramato, usado por 33 mulheres isoladamente e por 3 mulheres em associação com outro fármaco profilático (Fig. 2).

Não se verificou associação entre o fármaco utilizado ou o número de fármacos usados e o tipo de enxaqueca (com aura versus sem aura) ( $p=0,879$ e $p=0,529$ respetivamente) nem com o momento de ocorrência das crises em relação ao ciclo menstrual $(p=0,979$ e $p=0,683$ respetivamente).

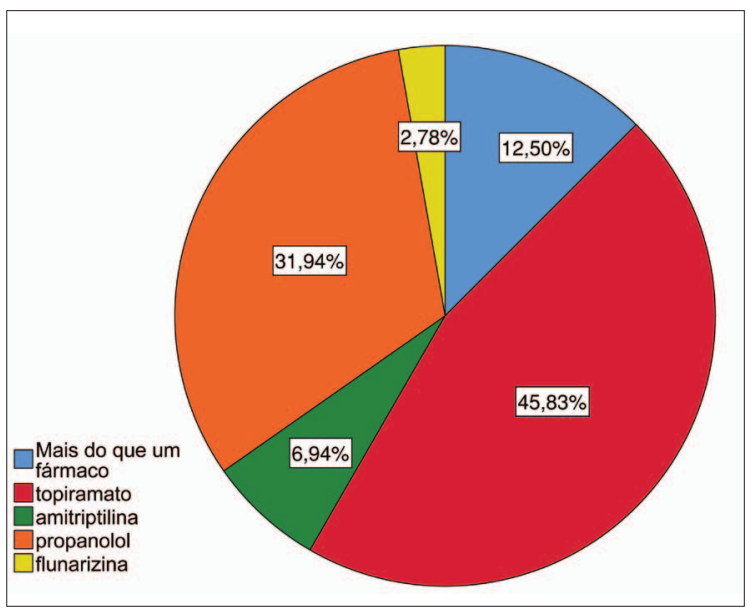

Figura 2. Uso de medicação profilática.

\section{Uso de outros métodos contracetivos no passado}

Da amostra total do estudo, $75 \%(n=8 I)$ reportou ter utilizado, no passado, um método contracetivo diferente do atual. Relativamente a este uso passado, não se verificou diferença estatisticamente significativa entre os grupos com aura versus sem aura $(p=0,374)$ ou entre os grupos definidos pelo momento de ocorrência das crises de enxaqueca relativamente ao cataménio $(p=0,34 \mathrm{I})$.

Das mulheres que já tinham experimentado mudança de método contracetivo $(n=8 I), 38 \%$ referiu que a mudança de método contracetivo alterou as características (em intensidade e/ou frequência) da enxaqueca. Esta noção de alteração bem como o sentido dessa alteração (melhorar ou piorar) não foi estatisticamente diferente nos grupos da enxaqueca com aura versus sem aura ( $p=0,393$ e $p=0,923$, respetivamente).
É de notar que das mulheres que reportaram alteração das características da enxaqueca, nenhuma pertencia ao grupo das mulheres cujas crises de enxaqueca ocorrem sempre fora do cataménio. De entre as mulheres que se recordavam de alguma alteração das características (intensidade/frequência) da enxaqueca $(n=31)$, 15 referiram melhoria das crises ao trocar a $\mathrm{COC}$ por contraceção não hormonal e duas referiram agravamento ao mudar de COC para contraceção não hormonal; quatro referiram agravamento ao passar de método não hormonal para $\mathrm{COC}$ e uma doente referiu melhoria na frequência das crises ao passar de método não hormonal para COC.

Em relação ao uso de métodos progestativos exclusivos, uma doente referiu que passar de um método não hormonal para implante subcutâneo com progestativo piorou as crises de enxaqueca; cinco doentes referiram que passar de $\mathrm{COC}$ para método progestativo exclusivo melhorou as crises e um referiu que a passagem de pílula progestativa para $\mathrm{COC}$ piorou.

No que respeita ao impacto da troca entre COC $(5$ opiniões) observou-se heterogeneidade nas respostas, mas os diferentes progestativos envolvidos e as diferentes doses de etinilestradiol utilizadas, não permitiram estabelecer um padrão.

As impressões subjetivas aqui reportadas não estão associadas ao tipo de enxaqueca nem ao tipo de predominância temporal da crise $(p=0,923$ e $p=0,697)$.

\section{Discussão}

Apesar deste estudo incluir apenas mulheres com enxaqueca, a prevalência do uso de cada método contracetivo encontrada está em linha com os dados apresentados por um estudo realizado em Portugal, em 2015, representativo das mulheres portuguesas. ${ }^{13} \mathrm{Em}$ ambos os trabalhos, os métodos contracetivos orais assumem-se como os mais prevalentes (usados por mais de $50 \%$ das mulheres em estudo).

Quer o Consenso sobre Contraceção da Sociedade Portuguesa de Ginecologia, ${ }^{9}$ quer os critérios médicos de elegibilidade para o uso dos métodos contracetivos da Organização Mundial de Saúde ${ }^{10}$ apresentam a COC como contraindicada na mulher com enxaqueca com aura e na mulher com enxaqueca sem aura e 35 ou mais anos de idade. A proporção, que foi encontrada neste estudo, de mulheres em que não estão a ser cumpridas as recomendações relativas à escolha do método con- 
tracetivo é elevada $(30,5 \%)$ e deve deixar não apenas os neurologistas, mas também os ginecologistas e os médicos de medicina geral e familiar alertados para as questões do planeamento familiar e prevenção cardiovascular em mulheres em idade fértil.

Contudo, a população deste estudo é composta por mulheres observadas numa consulta hospitalar de cefaleias em 2019, podendo o ingresso no estudo ter ocorrido quer em primeiras consultas quer em consultas de seguimento. Assim, nomeadamente no caso das mulheres que já se encontravam em seguimento, é possível que já thes tivesse sido explicado o aumento do risco vascular relacionado com o uso de $\mathrm{COC}$ e recomendada modificação do método ou encaminhamento para consulta de planeamento familiar. Assim, os resultados encontrados em relação ao não cumprimento das recomendações podem dever-se à falta de aconselhamento e promoção da modificação do método contracetivo em uso ou à preferência informada da mulher.

Verificou-se que 18 das 108 mulheres não utilizavam qualquer método contracetivo. Porém, não tendo sido explorado o motivo desta não utilização (abstinência sexual, infertilidade, menopausa, outra), algumas das mulheres que referiram não usar nenhum método contracetivo, podem fazê-lo porque estão na menopausa. Contudo, do mesmo modo, mulheres que usem contracetivo hormonal combinado $(\mathrm{CHC})$ podem manter-se com hemorragia de privação na semana de pausa, considerando que se encontram na idade fértil quando, na realidade, já não se encontram com cataménios espontâneos caso suspendessem o $\mathrm{CHC}$.

Dado que a enxaqueca sem aura é mais prevalente $\mathrm{e}^{14,15}$ e reportada como tal mesmo em amostras acompanhadas em consultas hospitalares especializadas ou centros de referência, ${ }^{16-18} \circ$ facto de não observarmos esta maior prevalência na amostra estudada poderá ser explicado por um viés de referenciação à CECef, não refletindo assim as doentes acompanhadas em CECef a distribuição na população geral. Embora possa haver tendência para que a prevalência da enxaqueca com aura seja subestimada (a aura pode ser difícil de identificar e descrever, mesmo em entrevista dirigida, entre outros motivos), ${ }^{19}$ o receio dos doentes e dos médicos não neurologistas decorrente das manifestações de aura e da maior associação entre enxaqueca com aura e eventos vasculares ${ }^{5}$ poderão levar a uma maior referenciação destas doentes à CECef em detrimento de doen- tes com enxaqueca sem aura que vão sendo geridos em consultas de cuidados de saúde primários, por exemplo.

Embora não tenha sido verificada uma diferença estatisticamente significativa entre o tipo de enxaqueca (com versus sem aura) nas mulheres que referiram enxaqueca associada ao cataménio, está descrito que as enxaquecas menstruais puras são tipicamente sem aura mesmo que as doentes apresentem enxaqueca com aura noutros momentos do ciclo menstrual. ${ }^{15,20}$ Assim, o resultado encontrado poder-se-á dever ao facto de não ter sido apurado junto das mulheres com enxaqueca com aura se todas as crises habitualmente cursavam ou não com aura bem como de se ter incluído no mesmo subgrupo de análise as mulheres cujas crises ocorrem apenas durante o cataménio e as mulheres cujas crises ocorrem predominantemente no cataménio.

Para além disso, a relação das crises de enxaqueca com o cataménio foi avaliada por resposta das mulheres a um inquérito e não através de um calendário de cataménios e cefaleias que permitisse aos investigadores objetivar a real relação - assim, este pode constituir um viés neste trabalho.

A ocorrência de enxaqueca está descrita como frequentemente associada ao ciclo menstrual. Uma revisão publicada em $2015^{15}$ e um estudo longitudinal também publicado em $2015^{21}$ mostraram que mais de $50 \%$ das mulheres refere ter uma enxaqueca associada ao cataménio (as crises ocorrem comumente associadas ao cataménio, mas também noutras alturas do ciclo menstrual) sendo que na nossa amostra o valor foi também ele superior a $50 \%(76,9 \%)$. A enxaqueca que ocorre apenas ou predominantemente associada ao cataménio foi identificada em $18,5 \%$ da amostra o que vai de encontro ao facto da enxaqueca menstrual ser citada como menos frequente $\left(<10 \%{ }^{15}\right.$ e $\left.5,5 \%^{21}\right)$. Contudo, a maior disparidade entre os dados encontrados e os já descritos prende-se com a prevalência da enxaqueca não relacionada com o cataménio, que na amostra representou apenas em $4,6 \%$ das mulheres e existem descrições que chegam a $40 \% .{ }^{21}$ Esta disparidade poderá ser explicada pela reduzida dimensão da amostra e/ ou por eventual viés de memória das doentes aquando da identificação da relação temporal entre as crises de enxaqueca e o cataménio.

Apesar de na nossa amostra a impressão subjetiva de melhoria ou agravamento da enxaqueca com alteração do método contracetivo não estar associada ao tipo de 
enxaqueca nem à relação temporal entre a enxaqueca e o cataménio, existe uma relação entre a enxaqueca e as hormonas sexuais (endógenas ou exógenas). A literatura sugere que o surgimento de crises de enxaqueca no período do cataménio se deve a uma queda dos níveis séricos de estrogénio na fase final do ciclo menstrual. ${ }^{20,22}$ Esta relação permite que o uso de determinado contracetivo se possa associar a agravamento ou melhoria do número e/ou intensidade das crises de enxaqueca. ${ }^{23}$

A literatura descreve que os efeitos da introdução de contraceção estroprogestativa em mulheres com enxaqueca podem ser muito heterogéneos ${ }^{15}$ tal como foi verificado na nossa amostra. Existe evidência de que o uso contínuo de estroprogestativos poderá associar-se a redução do número e/ou intensidade das crises de enxaqueca. ${ }^{24,25}$ Contudo, neste estudo não foi avaliada a posologia (intermitente ou contínua) de toma da COC nas mulheres que a utilizavam.

Em relação ao impacto do uso de contraceção exclusivamente progestativa, na amostra em estudo verificou-se que 6 de 7 mulheres reportaram melhoria do número e/ou intensidade das crises de enxaqueca com o uso do progestativo, dados que estão de acordo com estudos que mostram quer a diminuição quer o não aumento do número de crises com o uso de contracetivo progestativo, ${ }^{15,24}$ ainda que se saiba que a contraceção progestativa não causa supressão ovárica suficiente para ter impacto na ocorrência das crises de enxaqueca menstrual. ${ }^{24}$

Ainda em relação ao impacto do uso do método contracetivo nas crises de enxaqueca, é de salientar a possibilidade de viés de memória por parte das doentes quando reportam a sua impressão subjetiva.

\section{Conclusão}

Verificou-se uma prevalência anormal de enxaqueca com aura na amostra em estudo. Os métodos contracetivos orais assumem-se como os mais usados pelas mulheres. A proporção, encontrada neste estudo, de mulheres em que não estão a ser cumpridas as recomendações relativas à escolha do método contracetivo é elevada. Para se compreender melhor o impacto do uso dos diversos métodos contracetivos nas crises de enxaqueca são necessários estudos com um maior número de utentes e com metodologia capaz de objetivar de forma mais fidedigna a intensidade e frequência das crises.

\section{Responsabilidades Éticas}

Conflitos de Interesse: Os autores declaram a inexistência de conflitos de interesse na realização do presente trabalho.

Fontes de Financiamento: Não existiram fontes externas de financiamento para a realização deste artigo.

Confidencialidade dos Dados: Os autores declaram ter seguido os protocolos da sua instituição acerca da publicação dos dados de doentes.

Proteção de Pessoas e Animais: Os autores declaram que os procedimentos seguidos estavam de acordo com os regulamentos estabelecidos pelos responsáveis da Comissão de Investigação Clínica e Ética e de acordo com a Declaração de Helsínquia revista em 2013 e da Associação Médica Mundial.

Proveniência e Revisão por Pares: Não comissionado; revisão externa por pares.

Ethical Disclosures

Conflicts of Interest: The authors have no conflicts of interest to declare.

Financing Support: This work has not received any contribution, grant or scholarship

Confidentiality of Data: The authors declare that they have followed the protocols of their work center on the publication of data from patients.

Protection of Human and Animal Subjects: The authors declare that the procedures followed were in accordance with the regulations of the relevant clinical research ethics committee and with those of the Code of Ethics of the World Medical Association (Declaration of Helsinki as revised in 2013).

Provenance and Peer Review: Not commissioned; externally peer reviewed.

\section{References / Referências}

1. Vos T, Flaxman AD, Naghavi M, Lozano R, Michaud C, Ezzati $\mathrm{M}$, et al. Years lived with disability (YLDs) for 1160 sequelae of 289 diseases and injuries 1990-2010: a systematic analysis for the Global Burden of Disease Study 2010. Lancet. 2012;380:2163-96. doi: 10.1016/S0140-6736(12)61729-2.

2. Monteiro JM, Matos E, Calheiros JM. Headaches in Medical School Students. Neuroepidemiology. 1993;13:103-7. doi: 10.1159/000110366

3. Monteiro JM. Cefaleias: Estudo epidemiológico e clínico de uma população urbana. [Dissertação de Doutoramento]. Porto: ICBAS - Universidade do Porto; 1995.

4. Burch RC, Loder S, Loder E, Smitherman TA. The prevalence and burden of migraine and severe headache in the United States: updated statistics from government health surveillance studies. Headache 2015;55:21-34. doi: 10.1111/head.12482.

5. Schürks $M$, Rist PM, Bigal ME, Buring JE, Lipton RB, Kurth $T$, et al. Migraine and cardiovascular disease: systematic review and meta-analysis. BMJ. 2009;339:b3914. doi:10.1136/bmj.b3914

6. Hippisley-Cox J, Coupland C, Brindle P. Development and validation of QRISK3 risk prediction algorithms to estimate future risk of cardiovascular disease: prospective cohort study. BMJ. 2017;357:j2099. doi: 10.1136/bmj.j2099.

7. Gynecologists TACoOa. Womens Health Care Physicans. Frequently Asked Questions - FAQ 185 - Contraception Combined Hormonal Birth Control. ACOG 2018. [acedido a 03 de dezembro de 2020]. Disponível em: https://www. acog.org/womens-health/faqs/combined-hormonal-birthcontrol-pill-patch-ring

8. Etminan M, Takkouche B, Isorna FC, Samii A. Risk of ischaemic stroke in people with migraine: systematic review and meta-analysis of observational studies. BMJ. 2005;330:63. doi: 10.1136/bmj.38302.504063.8F.

9. Sociedade Portuguesa Ginecologia SPC, Sociedade Portuguesa Medicina Reprodução. Consenso sobre contracepção. Estoril: Reunião de Consenso Nacional sobre Contracepção; 2011 
10. World Health Organization. Medical Eligibility Criteria for Contraceptive Use. 5th ed. Geneve: WHO; 2015.

11. Gold EB. The timing of the age at which natural menopause occurs. Obstet Gynecol Clin North Am. 2011;38:425-440. doi:10.1016/j.ogc.2011.05.002

12. Direção Geral da Saúde: Programa Nacional de Saúde Reprodutiva. Saúde reprodutiva/Planeamento familiar. Lisboa: DGS; 2008.

13. Águas $F$, Bombas $T$, Silva $D$. Avaliação das práticas contracetivas das mulheres em Portugal. Acta Obstét Ginecol Port. 2016;10:184-92.

14. Russell MB, Rasmussen BK, Thorvaldsen P, Olesen J. Prevalence and sex-ratio of the subtypes of migraine. Int J Epidemiol. 1995;24:612-8. doi: 10.1093/ije/24.3.612.

15. Cappy H, Lucas C, Catteau-Jonard S, Robin G. Migraine and contraception. Gynecol Obstet Fertil. 2015;43:234-41. doi: 10.1016/j.gyobfe.2015.01.006.

16. Diamond ML, Cady RK, Mao L, Biondi DM, Finlayson G, Greenberg SJ, et al. Characteristics of Migraine Attacks and Responses to Almotriptan Treatment: A Comparison of Menstrually Related and Nonmenstrually Related Migraines. Headache. 2008; 48:248-58. doi: 10.1111/j.15264610.2007.01019.x.

17. Rockett FC, Ada S, Perry ID, Chaves ML. Cardiovascular disease risk in women with migraine. J Headache Pain. 2013;14:75. doi:10.1186/1129-2377-14-75
18. Hansen JM, Goadsby PJ, Charles A. Reduced efficacy of sumatriptan in migraine with aura vs without aura. Neurology. 2015;84:1880-5. doi: 10.1212/WNL.0000000000001535.

19. Machado J, Barros J, Palmeira M. Enxaqueca: Fisiopatogenia, clínica e tratamento. Rev Port Med Geral Fam. 2006;22:461-70. doi: 10.32385/rpmgf.v22i4.10267.

20. Calhoun AH, Batur P. Combined hormonal contraceptives and migraine: An update on the evidence. Cleve Clin J Med 2017;84:631-8. doi: 10.3949/ccjm.84a.16033.

21. Pavlovic JM, Stewart WF, Bruce CA, Gorman JA, Sun H, Buse DC, et al. Burden of migraine related to menses: results from the AMPP study. J Headache Pain. 2015;16:24. doi: 10.1186/s10194-015-0503-y.

22. MacGregor EA. Estrogen and Migraine: Correlations and Prevention. Headache. 2008;48:S99-S107. doi: 10.1111/j.1526-4610.2008.01202.x

23. Nappi RE, Merki-Feld GS, Terreno E, Pellegrinelli A, Viana M. Hormonal contraception in women with migraine: is progestogen-only contraception a better choice? J Headache Pain. 2013;14:66. doi:10.1186/1129-2377-14-66

24. Harris $M$, Kaneshiro B. An evidence-based approach to hormonal contraception and headaches. Contraception. 2009; 80:417-21. doi: 10.1016/j.contraception.2009.04.014.

25. Chai NC, Peterlin BL, Calhoun AH. Migraine and estrogen. Curr Opin Neurol. 2014;27:315-24. doi: 10.1097/ WCO.0000000000000091. 